\title{
African Ancestry Gradient Is Associated with Lower Systemic $\mathbf{F}_{2}$-Isoprostane Levels
}

\author{
Francis Annor, ${ }^{1}$ Michael Goodman, ${ }^{2}$ Bharat Thyagarajan, ${ }^{3}$ Ike Okosun, 1 \\ Ayo Doumatey, ${ }^{4}$ Barbara A. Gower, ${ }^{5}$ and Dora Il'yasova ${ }^{1}$ \\ ${ }^{1}$ Division of Epidemiology and Biostatistics, School of Public Health, Georgia State University, One Park Place, Suite 630, \\ Atlanta, GA 30303, USA \\ ${ }^{2}$ Department of Epidemiology, Rollins School of Public Health, Emory University, 1518 Clifton Road, Atlanta, GA 30322, USA \\ ${ }^{3}$ Department of Laboratory Medicine and Pathology, University of Minnesota, 420 Delaware St SE 6435, Minneapolis, \\ MN 55455, USA \\ ${ }^{4}$ Center for Research on Genomics and Global Health, National Human Genome Research Institute, National Institutes of Health, \\ Building 12A, Room 4047, 12 South Dr, MSC 5635, Bethesda, MD 20892, USA \\ ${ }^{5}$ Department of Nutrition Sciences, University of Alabama at Birmingham, 1675 University Blvd., Birmingham, AL 35294, USA
}

Correspondence should be addressed to Dora Il'yasova; dilyasova@gsu.edu

Received 7 November 2016; Accepted 4 January 2017; Published 31 January 2017

Academic Editor: Cinzia Signorini

Copyright (c) 2017 Francis Annor et al. This is an open access article distributed under the Creative Commons Attribution License, which permits unrestricted use, distribution, and reproduction in any medium, provided the original work is properly cited.

\begin{abstract}
Context. Low levels of systemic $\mathrm{F}_{2}$-isoprostanes $\left(\mathrm{F}_{2}\right.$-IsoP) increase the risk of diabetes and weight gain and were found in African Americans. Low $\mathrm{F}_{2}$-IsoPs could reflect an unfavorable metabolic characteristic, namely, slow mitochondrial metabolism in individuals with African ancestry. Objective. To examine differences in plasma $\mathrm{F}_{2}$-IsoPs in three groups with a priori different proportion of African ancestry: non-Hispanic Whites (NHWs), US-born African Americans (AAs), and West African immigrants (WAI). Design. Cross-sectional study. Setting. Georgia residents recruited from church communities. Participants. 218 males and females 25-74 years of age, who are self-identified as NHW $(n=83)$, AA $(n=56)$, or WAI $(n=79)$. Main Outcome Measure(s). Plasma $\mathrm{F}_{2}$-IsoPs quantified by gas chromatography-mass spectrometry. Results. After adjustment for age, gender, obesity, and other comorbidities, WAI had lower levels of plasma $\mathrm{F}_{2}$-IsoP than AA (beta-coefficient $=-9.8, p<0.001$ ) and AA had lower levels than NHW (beta-coefficient $=-30.3, p<0.001$ ). Similarly, among healthy nonobese participants, $\mathrm{F}_{2}$-IsoP levels were lowest among WAI, followed by AA, and the highest levels were among NHW. Conclusion. Plasma $\mathrm{F}_{2}$-IsoPs are inversely associated with African ancestry gradient. Additional studies are required to test whether optimization of systemic $\mathrm{F}_{2}$-IsoP levels can serve as means to improve race-specific lifestyle and pharmacological intervention targeted to obesity prevention and treatment.
\end{abstract}

\section{Introduction}

Metabolic predisposition to obesity and type 2 diabetes among African Americans is well-established [1-3]. Identification of modifiable factors that predispose African Americans to these metabolic disorders may offer opportunities for targeted intervention. The importance of targeting racespecific metabolic predisposition is emphasized by the findings from weight loss interventions, showing that with similar treatments whether lifestyle-focused $[2,4,5]$ or surgical $[6,7]$, African Americans tend to lose less weight than European Americans.
Our previous work examined the relationships between urinary $\mathrm{F}_{2}$-isoprostanes ( $\mathrm{F}_{2}$-IsoPs) as validated measures of oxidative status and the risk of weight gain [8] and type 2 diabetes [9]. Despite the conventional point of view that elevated $\mathrm{F}_{2}$-IsoP level reflects harmful oxidative stress [10, 11], we found that elevated $\mathrm{F}_{2}$-IsoP levels predicted lower risks of both weight gain [8] and type 2 diabetes [9]. Compared to Whites, African Americans have lower levels of urinary $\mathrm{F}_{2}$-IsoPs $[8,12]$ and this gap increases with obesity [12]. To explain these findings, we proposed a relationship between $\mathrm{F}_{2}$-IsoP levels and the intensity of mitochondrial oxidative metabolism. We reasoned that (a) $\mathrm{F}_{2}$-IsoPs are validated 
measures of the overall levels of reactive oxygen species (ROS) $[13,14]$ and (b) mitochondrial oxidative metabolism is the major endogenous source of ROS [15]. Thus, low $\mathrm{F}_{2}$ IsoP levels could reflect a metabolic profile that is known to be prevalent in African Americans, that is, slower mitochondrial oxidative metabolism [16-19]. We further hypothesized that systemic $\mathrm{F}_{2}$-IsoP levels present a race-specific metabolic phenotype that is linked to African ancestry. To test this hypothesis, we compared systemic $\mathrm{F}_{2}$-IsoP levels across three groups with a priori different proportion of African ancestry: non-Hispanic Whites (NHWs), US-born African Americans (AAs), and West African immigrants (WAI). African Americans are genetically related to the West African ancestry [20]; however, the percentage of African ancestry in selfidentified AA is substantially lower (83\%) as compared to WAI (95\%) [21]. In this study, systemic $\mathrm{F}_{2}$-IsoP levels were measured in plasma. It has been established that both the urinary and the circulating levels of $\mathrm{F}_{2}$-IsoP present an accurate quantification of the endogenous production of these molecules [22]. We hypothesized that WAI, the group with the greatest proportion of African ancestry, will have the lowest systemic levels of $\mathrm{F}_{2}$-IsoPs among these three racial/ethnic groups, followed by AA.

\section{Materials and Methods}

2.1. Study Population. We used cross-sectional data from a previously conducted Study on Race, Stress, and Hypertension (SRSH). The study was designed to assess the differences in dietary, lifestyle, and psychosocial exposures in relation to blood pressure in a racially and ethnically diverse population. The methods of the study are described in detail elsewhere [23]. Briefly, the eligibility criteria included being an adult 25-74 years of age, self-identified as NHW, AA or WAI, and a permanent Georgia resident. For the WAI participants, an additional inclusion criterion was arrival to the US after the 18 th birthday. Subjects were excluded if they did not give informed consent. There were 335 individuals who met the initial study inclusion criteria. Of those, 117 participants were excluded from the analyses due to missing values for plasma $\mathrm{F}_{2}$-IsoPs. All methods were reviewed and approved by the Institutional Review Boards of the Emory University and the Georgia State University.

2.2. Measurements of Plasma $F_{2}$-IsoPs. All participants provided blood samples that were drawn into five $10 \mathrm{~mL}$ vacutainer tubes ( 2 sodium heparin tubes, 1 EDTA tube, and 2 red top tubes for serum collection) and immediately plunged into ice and protected from direct light. Plasma, serum, and buffy coat specimens were separated within $4-8$ hours by centrifugation under refrigeration, aliquoted, frozen, and stored at $-80^{\circ} \mathrm{C}$. The aliquots were then shipped overnight on dry ice for molecular analysis by the Molecular Epidemiology and Biomarker Research Laboratory (MEBRL) at the University of Minnesota, Minneapolis, MN. Gas chromatography-mass spectrometry (GCMS) [24] was used to measure plasma free $\mathrm{F}_{2}$-IsoPs. The $\mathrm{F}_{2}$-IsoP were extracted from the plasma sample and deuterium (4)-labeled 8-iso-prostaglandin $\mathrm{F}_{2}$-alpha was used as an internal standard. The CV for the F2-isoprostane measurements was $9.7 \%$ for the "low" control (mean value = $47.48 \mathrm{pg} / \mathrm{mL}$ ) and the CV for the "high" control is $11.2 \%$ (mean value $=89.87 \mathrm{pg} / \mathrm{mL}$ ).

2.3. Other Measurements. Self-administered questionnaire collected data on demographic characteristics (age, sex, race/origin, and education), medical history (hypertension and use of medications), and lifestyle (physical activity and smoking) for all participants. The questionnaires were filled out and returned during the data collection sessions. Blood pressure and anthropometric measures (height and weight) were also taken during data collection sessions. The reported and measured BMI were highly correlated $(r=0.91)$. Hypertension was determined based on the blood pressure measurements (conducted by trained and certified staff using a standardized protocol) and self-report. Self-reported history of chronic diseases was broadly categorized into the following: diseases of heart (angina, congestive heart failure, myocardial infarction, hypertension, high blood cholesterol, pulmonary embolism, stroke, and thrombophlebitis), kidney (chronic kidney disease and kidney stone), endocrine diseases (diabetes, hypoglycemia and overactive thyroid), cancer (melanoma, skin cancer, and other cancers), and allergies (hay fever and atopic dermatitis). Medical history and hypertension were summarized as morbidity score, which represents a number of conditions reported by the participants and detected hypertension.

2.4. Statistical Analysis. The main objective of this analysis was to examine the relationship between race/ethnicity and circulating $\mathrm{F}_{2}$-IsoPs. To screen for potential confounders, study characteristics were compared between the three racial/ethnic groups using chi-square and Kruskal-Wallis tests for categorical and continuous variables, respectively (Table 1). Similarly, crude associations between study characteristics and plasma $\mathrm{F}_{2}$-IsoPs were examined using chi-square and Kruskal-Wallis tests for categorical and continuous variables, respectively (Table 2 ). Smoking was not considered as a potential confounder in this study, because only $5 \%$ of the study population were current smokers and smoking was shown not to be associated with F2-IsoP levels [23]. Linear regression models were used to examine the influence of potential confounders on the relationship between race/ethnicity and plasma $\mathrm{F}_{2}$-IsoPs (Table 3). Race/ethnicity was coded as a categorical variable (NHW/AA/WAI) with AA being the reference category. Model 1 included variables associated with both the exposure (race/ethnicity) and the outcome (plasma $\mathrm{F}_{2}$-IsoPs), except morbidity index. Model 2 includes significant predictors of plasma $\mathrm{F}_{2}$-IsoPs from Model 1 and morbidity index. Model 3 includes a modified morbidity index that incorporates obesity as a morbidity condition. This analysis was conducted using both the original scale variable for $\mathrm{F}_{2}$-isoprostanes and natural log-transformed variable. The comparison of F2-isoprostane distribution between the racial/ethnic groups stratified by comorbidity score was conducted using Kruskal-Wallis tests. All analyses were performed in SAS statistical software version 9.3 (SAS Institute Inc, Cary, NC). 
TABLE 1: Characteristics of the study population.

\begin{tabular}{|c|c|c|c|c|}
\hline & $\begin{array}{c}\text { WAI }^{\mathrm{a}} \\
(n=79)\end{array}$ & $\begin{array}{c}\mathrm{AAs}^{\mathrm{a}} \\
(n=56)\end{array}$ & $\begin{array}{l}\text { NHWs }^{\mathrm{a}} \\
(n=83)\end{array}$ & $p$ value $^{\mathrm{b}}$ \\
\hline \multicolumn{5}{|l|}{ Variable } \\
\hline Mean age (SD), years & $42.5(10.9)$ & $49.1(11.8)$ & $48.2(14.0)$ & 0.002 \\
\hline Sex, \% males & 37.8 & 51.9 & 33.7 & 0.10 \\
\hline Education, \% college graduates & 52.0 & 32.1 & 43.9 & 0.04 \\
\hline $\mathrm{BMI}(\mathrm{SD}), \mathrm{kg} / \mathrm{m}^{2}$ & $28.7(6.7)$ & $31.0(6.7)$ & $30.1(6.5)$ & 0.21 \\
\hline$\%$ Obese & 39.7 & 50.0 & 42.2 & 0.50 \\
\hline \multicolumn{5}{|l|}{ Medical history (Yes \%) } \\
\hline Heart diseases & 21.3 & 57.5 & 52.5 & $<0.001$ \\
\hline Kidney diseases & 0.0 & 9.1 & 15.3 & 0.001 \\
\hline Endocrine diseases & 5.4 & 31.3 & 10.2 & $<0.001$ \\
\hline Cancer & 1.4 & 0.0 & 8.8 & 0.1138 \\
\hline Allergy & 9.6 & 33.3 & 38.6 & 0.003 \\
\hline \multicolumn{5}{|l|}{ Morbidity score, $\%$} \\
\hline 0 (number of categories reported) & 74.0 & 28.3 & 21.1 & \multirow{4}{*}{$<0.001$} \\
\hline 1 & 16.4 & 34.8 & 42.1 & \\
\hline 2 & 8.2 & 15.6 & 31.6 & \\
\hline$\geq 3$ & 1.4 & 21.9 & 5.3 & \\
\hline
\end{tabular}

${ }^{a}$ WAI: West African immigrants, AAs: African Americans, and NHWs: non-Hispanic Whites.

${ }^{\mathrm{b}} p$ values are presented for Chi-square test (categorical variables) and for Kruskal-Wallis rank sum test (continuous variables).

\section{Results}

WAI were on average younger and had greater level of education (Table 1). Importantly, WAI were also healthier as compared to AAs and NHWs, reporting in their medical history fewer health conditions (Table 1). BMI did not vary among the racial/ethnic groups ( $p$ value 0.2 ). Plasma $\mathrm{F}_{2}$-IsoP levels were inversely associated with African ancestry gradient and were lowest among WAI and highest among NHW (Table 2). We followed the conventional rule for confounder screening by examining the associations of different study characteristics with both the main exposure (race/ethnicity, Table 1$)$ and the outcome ( $\mathrm{F}_{2}$-IsoP levels, Table 2$)$. The data presented in Tables 1 and 2 indicate that age, BMI, and morbidity scores are potential confounders as these characteristics were associated with race/ethnicity and $\mathrm{F}_{2}$-IsoP levels. Obese individuals (BMI $\geq 30 \mathrm{~kg} / \mathrm{m}^{2}$ ) on average had $35 \%$ greater levels of $\mathrm{F}_{2}$-IsoPs $(p=0.04)$ (Table 2). Among the reported chronic conditions, heart and kidney diseases as well as allergies were associated with greater $\mathrm{F}_{2}$-IsoP levels $(p<0.01)$, whereas endocrine conditions and cancer showed a trend toward positive association ( $p$ values 0.09 and 0.07 , resp.). This analysis revealed two major determinants of plasma $\mathrm{F}_{2}$-IsoP levels in this study population: race/ethnicity and the overall comorbidity expressed as morbidity score.

The association between race/ethnicity and plasma $\mathrm{F}_{2}$ IsoP levels was examined by linear regression (Table 3). Age and sex were not significant predictors of $\mathrm{F}_{2}$-IsoP levels (Model 1, Table 3) and therefore were excluded from the final model. This exclusion did not substantially change the estimates of beta coefficients for race/ethnicity (data not shown). BMI was a significant predictor of $\mathrm{F}_{2}$-IsoP levels only in the absence of morbidity score (Models 1 and 2, Table 3). As BMI correlated with morbidity score $(r=0.53$, $p<0.001$ ), we included obesity into the morbidity score in the final model (Model 3, Table 3). Overall, WAI had the lowest levels of plasma $\mathrm{F}_{2}$-IsoP and $\mathrm{AA}$ had higher $\mathrm{F}_{2}$-IsoP levels as compared to WAI but lower than NHW (Table 3). When stratified by morbidity score that included obesity, $\mathrm{F}_{2}$ IsoP levels were lowest among WAI, followed by $\mathrm{AA}$, and the highest levels were among NHW among healthy nonobese participants as well as among participants with comorbidities (Figure 1). Kruskal-Wallis showed significant differences across the groups within each comorbidity category ( $p$ values $<0.001)$.

\section{Discussion}

The main finding of this study is the inverse relationship between African ancestry gradient and systemic $\mathrm{F}_{2}$-IsoP levels. The observed association was statistically significant and in the hypothesized direction. This inverse relationship is confirmed by both the crude and regression analyses, after adjustment for potential confounders. Specifically, age, gender, BMI, and comorbidities were examined as potential confounders based on the previous studies showing associations between these variables and either race or systemic $\mathrm{F}_{2}$-IsoPs levels. The stratified analysis demonstrated that, among healthy nonobese participants, the gradient of African ancestry inversely correlates with plasma $\mathrm{F}_{2}$-IsoP levels. Stratification by obesity and comorbidity status may be important, because the differences in plasma $\mathrm{F}_{2}$-IsoP levels 
TABLE 2: Relationships between plasma $\mathrm{F}_{2}$-isoprostanes and study variables.

\begin{tabular}{|c|c|c|}
\hline $\begin{array}{l}\text { Continuous/ordinal } \\
\text { variables }\end{array}$ & $\begin{array}{c}\text { Spearman correlation } \\
\text { coefficient }\end{array}$ & $p$ value \\
\hline Age & 0.148 & 0.03 \\
\hline $\mathrm{BMI}$ & 0.189 & $<0.01$ \\
\hline Morbidity score $^{\mathrm{a}}$ & 0.437 & $<0.01$ \\
\hline $\begin{array}{l}\text { Categorical variables } \\
\text { (Kruskal-Wallis test) }\end{array}$ & $\begin{array}{c}\mathrm{F}_{2} \text {-isoprostane level mean } \\
(\mathrm{SD}) \\
(\mathrm{pg} / \mathrm{mL})\end{array}$ & $p$ value \\
\hline \multicolumn{3}{|l|}{ Race } \\
\hline NHW & $80.1(34.9)$ & \multirow{2}{*}{$<0.001$} \\
\hline $\mathrm{AA}$ & $51.1(20.1)$ & \\
\hline NHW & $80.1(34.9)$ & \multirow{2}{*}{$<0.001$} \\
\hline WAI & $33.8(9.0)$ & \\
\hline $\mathrm{AA}$ & $51.1(20.1)$ & \multirow{2}{*}{$<0.001$} \\
\hline WAI & $33.8(9.0)$ & \\
\hline \multicolumn{3}{|l|}{ Sex } \\
\hline Females & $59.4(35.8)$ & \multirow{2}{*}{0.43} \\
\hline Males & $51.1(23.2)$ & \\
\hline \multicolumn{3}{|l|}{ Education } \\
\hline College graduate & $50.5(24.8)$ & \multirow{4}{*}{0.26} \\
\hline Some college & $59.2(35.2)$ & \\
\hline HS grads & $60.9(30.1)$ & \\
\hline Less than HS & $53.8(31.0)$ & \\
\hline \multicolumn{3}{|l|}{ Obesity } \\
\hline Nonobese & $51.1(23.2)$ & \multirow{2}{*}{0.04} \\
\hline Obese & $69.2(35.8)$ & \\
\hline \multicolumn{3}{|l|}{ Heart disease } \\
\hline Yes & $58.6(30.1)$ & \multirow{2}{*}{$<0.01$} \\
\hline No & $47.0(24.9)$ & \\
\hline \multicolumn{3}{|l|}{ Endocrine disease } \\
\hline Yes & $56.6(23.9)$ & \multirow{2}{*}{0.09} \\
\hline No & $50.8(28.3)$ & \\
\hline \multicolumn{3}{|l|}{ Kidney disease } \\
\hline Yes & $82.6(32.3)$ & \multirow{2}{*}{$<0.01$} \\
\hline No & $49.3(25.9)$ & \\
\hline \multicolumn{3}{|l|}{ Allergy conditions } \\
\hline Yes & $67.0(28.4)$ & \multirow{2}{*}{$<0.01$} \\
\hline No & $46.4(25.6)$ & \\
\hline \multicolumn{3}{|l|}{ Cancer } \\
\hline Yes & $79.7(45.0)$ & \multirow{2}{*}{0.07} \\
\hline No & $50.3(26.4)$ & \\
\hline
\end{tabular}

${ }^{a}$ Morbidity score summarizes the number of comorbidities.

could be attributable to accumulated chronic conditions [10]. The observations that nonobese healthy individuals differ with respect to their plasma $\mathrm{F}_{2}$-IsoP levels and that the difference persists at greater comorbidity levels suggest that these differences reflect genetic variability rather than life

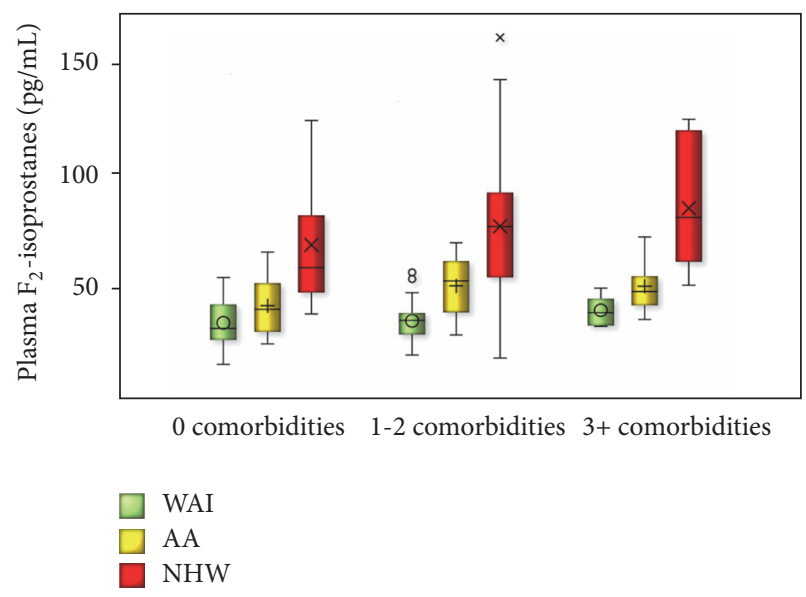

FIgURE 1: Distribution of plasma $\mathrm{F}_{2}$-isoprostanes in West African Immigrants (WAI), African Americans (AAs), and non-Hispanic Whites (NHWs) stratified by morbidity score. Boxplots are presented for each group, showing minimum, first quartile, median, third quartile, maximum, and the outliers (depicted as "o" and " $\mathrm{x}$ "). The $p$ values for comparison of F2-isoprostane levels between racial/ethnic groups are $<0.05$ within each strata of comorbidity score (Kruskal-Wallis tests).

style or health status. In essence, we propose that systemic $\mathrm{F}_{2}$ IsoP levels present a phenotypical characteristic that similarly to metabolism and obesity is influenced by genetic background but can be modified by lifestyle or pharmacological interventions. Below we discuss the biological meaning of systemic $\mathrm{F}_{2}$-IsoPs levels and a potential for intervention.

As systemic levels of $\mathrm{F}_{2}$-IsoPs are validated indices of oxidative status $[13,14]$, the greater levels of these compounds in circulation and in urine have been interpreted as indicators of harmful oxidative stress. This point of view draws on multiple findings of elevated systemic $\mathrm{F}_{2}$-IsoP levels in patients with different chronic conditions [10]. In this study, we also found that plasma $\mathrm{F}_{2}$-IsoP levels correlate with comorbidity index. However, these correlations may reflect the consequence rather than a cause of health problems, because prospective evidence does not support the associations between elevated $\mathrm{F}_{2}$-IsoP levels and onset of chronic diseases in three independent cohorts $[8,9,25-27]$. Moreover, physical activity, which is a known preventive factor for obesity and diabetes [28], leads to increased levels of systemic $\mathrm{F}_{2}$-IsoPs $[29,30]$. Thus, there is an apparent contradiction between the conventional interpretation of systemic $\mathrm{F}_{2}$-IsoP levels as oxidative stress and epidemiological evidence from prospective studies and studies of physical activity portraying these biomarkers as a favorable phenotype.

We proposed that the molecular mechanisms underlying individual differences in systemic $\mathrm{F}_{2}$-IsoP levels as markers of ROS generation are human variability in oxidative metabolism [31], which is the major endogenous source of ROS [15]. Several of our previously published findings indicate that systemic $\mathrm{F}_{2}$-IsoP levels may specifically reflect the intensity of mitochondrial fatty acid oxidation [30,32]. We demonstrated that urinary $\mathrm{F}_{2}$-IsoP levels are positively associated with the acylcarnitine species that are increased 
TABLE 3: Association between plasma $\mathrm{F}_{2}$-isoprostanes and African ancestry gradient.

\begin{tabular}{|c|c|c|c|c|c|c|}
\hline & \multicolumn{6}{|c|}{ Beta coefficient (SE) } \\
\hline & \multirow{2}{*}{\multicolumn{2}{|c|}{ Model 1}} & \multirow{2}{*}{\multicolumn{2}{|c|}{$\begin{array}{l}p \text { value } \\
\text { Model } 2\end{array}$}} & \multirow{2}{*}{\multicolumn{2}{|c|}{ Model $3^{\mathrm{b}}$}} \\
\hline & & & & & & \\
\hline & $\mathrm{A}^{\mathrm{a}}$ & $\mathrm{B}^{\mathrm{a}}$ & A & B & A & B \\
\hline \multirow{2}{*}{ AA (versus NHW) } & $-29.1(4.3)$ & $-0.436(0.066)$ & $-30.9(4.4)$ & $-0.468(0.076)$ & $-30.3(4.4)$ & $-0.456(0.076)$ \\
\hline & $<0.001$ & $<0.001$ & $<0.001$ & $<0.0001$ & $<0.001$ & $<0.0001$ \\
\hline \multirow{2}{*}{ AA (versus WAI) } & $15.8(4.5)$ & $0.349(0.069)$ & $9.8(4.5)$ & $0.263(0.077)$ & $9.8(4.5)$ & $-0.265(0.077)$ \\
\hline & 0.001 & $<0.001$ & 0.03 & 0.0008 & 0.03 & 0.0007 \\
\hline \multirow{2}{*}{ Age (years) } & $-0.06(0.1)$ & $-0.001(0.002)$ & & & & \\
\hline & 0.65 & 0.69 & & & & \\
\hline \multirow{2}{*}{ Sex (males versus females) } & $-5.3(3.5)$ & $-0.048(0.054)$ & & & & \\
\hline & 0.13 & 0.37 & & & & \\
\hline \multirow{2}{*}{ BMI $\left(\mathrm{kg} / \mathrm{m}^{2}\right)$} & $1.02(0.3)$ & $0.016(0.004)$ & $0.2(0.3)$ & $0.006(0.005)$ & \multirow{2}{*}{ Excluded } & \multirow{2}{*}{ Excluded } \\
\hline & $<0.001$ & 0.0001 & 0.40 & 0.22 & & \\
\hline \multirow{2}{*}{ Morbidity score } & & & $3.15(1.7)$ & $0.051(0.027)$ & $3.9(1.5)^{b}$ & $0.070(0.026)^{\mathrm{b}}$ \\
\hline & & & 0.07 & 0.06 & 0.01 & 0.007 \\
\hline
\end{tabular}

${ }^{\mathrm{a}} \mathrm{F}_{2}$-isoprostane variable presents the measurements on the original scale in $\mathrm{A}$ and on the natural log-transformed scale in $\mathrm{B}$.

${ }^{\mathrm{b}}$ In Model 3, obesity (BMI $\left.\geq 30\right)$ was included in morbidity index.

at the physiological state with enhanced fat oxidation [32] and that their basal level increases after aerobic training [30]. Taking into account that AA have lower levels of fat oxidation $[18,33]$ and mitochondrial metabolism [16-19], we propose that these phenotypes are associated with African ancestry and are reflected by low $\mathrm{F}_{2}$-IsoP levels. Other findings support the notion that genetic background might be connected to systemic $\mathrm{F}_{2}$-IsoP levels via intensified or slow metabolic phenotype. For example, children with sickle cell disease have hyperactive metabolism, which is associated with greater $\mathrm{F}_{2}$-IsoP levels [34, 35]. In contrast, patients with Down syndrome have lower metabolic rate and are prone to obesity [36], which corresponds to our findings of a trend of lower urinary $\mathrm{F}_{2}$-IsoP levels in Down syndrome patients [37]. On the other hand, in a relatively homogenous ethnic population of Danish monozygotic and dizygotic twins (with similar genetic background), the heritability of urinary $\mathrm{F}_{2}-$ IsoP levels was low $(\sim 22 \%)$ [38]. The Danish study suggests that systemic $\mathrm{F}_{2}$-IsoP levels can be a modifiable factor, corroborating our hypothesis that systemic $\mathrm{F}_{2}$-IsoP levels are related to mitochondrial oxidative metabolism, a metabolic characteristic that is flexible and can be targeted by lifestyle and/or pharmacological interventions [39-41].

The potential for interventions in addressing low mitochondrial metabolism and muscle fat utilization has been demonstrated by small studies showing that these metabolic traits can be reversed. For example, a study by Cortright et al. [18] showed that lean AA women had lower levels of muscle fat oxidation as compared to White women and that exercise training had a stronger effect on fat oxidation in AA women than in White women, suggesting that fat oxidation could be an effective target for race-specific interventions. Another example is a study by Williams et al. [42] demonstrating that metformin, a pharmacological agent known to increase fat oxidation, produced a two times greater reduction in HbAlc in diabetic African American compared $(n=7429)$ to White patients $(n=8783)$. However, the obvious barrier in assessing interventions that target mitochondrial metabolism and fat oxidation is absence of noninvasive means to monitor these phenotypical characteristics. Currently, muscle mitochondrial metabolism is assessed through muscle biopsy, which is invasive and cannot be applied for practical reasons. Similarly, the assessment of fat utilization by indirect calorimetry is time- and labor-consuming and also not applicable to population-based interventions. In contrast, systemic $\mathrm{F}_{2}$ IsoP levels can serve as a simple, noninvasive means of assessing these metabolic characteristics over time and would be widely applicable in both the research and clinical setting.

The important caveat, however, is the interpretation of systemic $\mathrm{F}_{2}$-IsoPs in weight loss interventions. As this study demonstrated and it also has been previously found in other populations [10], systemic $\mathrm{F}_{2}$-IsoP levels are associated with BMI. Moreover, weight loss, a desirable outcome, is associated with decrease in $\mathrm{F}_{2}$-IsoP levels [43]. This is in parallel with a known phenomenon of metabolic adaptation also known as adaptive thermogenesis, that is, compensatory changes in energy expenditure and fat oxidation that favor fat mass homeostasis [44]. It has been demonstrated that weight gain or loss leads to corresponding changes in energy expenditure and fat oxidation [33]. It is also known that metabolic response to weight perturbations differs between the individuals and is influenced by genetic background [45]. Currently, such metabolic adaptation favoring homeostasis of fat mass is one of the central problems in maintenance of weight loss [46]. Based on the phenomenon of metabolic adaptation, greater $\mathrm{F}_{2}$-IsoP levels should be used as a favorable phenotype only in relation to body fat mass or BMI. Such $\mathrm{F}_{2}$-IsoP/BMI index presents a noninvasive measure and may provide the basis for targeted interventions to prevent obesity and type 2 diabetes among populations of African descent. 


\section{Competing Interests}

The authors declare that there is no conflict of interests regarding the publication of this paper.

\section{References}

[1] A. E. Staiano, D. M. Harrington, N. M. Johannsen et al., "Uncovering physiological mechanisms for health disparities in type 2 diabetes," Ethnicity and Disease, vol. 25, no. 1, pp. 31-37, 2015.

[2] S. K. Kumanyika, M. C. Whitt-Glover, and D. Haire-Joshu, "What works for obesity prevention and treatment in black Americans? Research directions," Obesity Reviews, vol. 15, supplement 4, pp. 204-212, 2014.

[3] S. A. Black, "Diabetes, diversity, and disparity: what do we do with the evidence?" American Journal of Public Health, vol. 92, no. 4, pp. 543-548, 2002.

[4] K. H. Morales, S. K. Kumanyika, J. E. Fassbender, J. Good, A. R. Localio, and T. A. Wadden, "Patterns of weight change in black Americans: pooled analysis from three behavioral weight loss trials," Obesity, vol. 22, no. 12, pp. 2632-2640, 2014.

[5] M. L. Fitzgibbon, L. M. Tussing-Humphreys, J. S. Porter, I. K. Martin, A. Odoms-Young, and L. K. Sharp, "Weight loss and African-American women: a systematic review of the behavioural weight loss intervention literature," Obesity Reviews, vol. 13, no. 3, pp. 193-213, 2012.

[6] K. J. Coleman, Y.-C. Huang, F. Hendee, H. L. Watson, R. A. Casillas, and J. Brookey, "Three-year weight outcomes from a bariatric surgery registry in a large integrated healthcare system," Surgery for Obesity and Related Diseases, vol. 10, no. 3, pp. 396-403, 2014.

[7] K. E. Limbach, K. Ashton, J. Merrell, and L. J. Heinberg, "Relative contribution of modifiable versus non-modifiable factors as predictors of racial variance in Roux-en-Y gastric bypass weight loss outcomes," Obesity Surgery, vol. 24, no. 8, pp. 1379-1385, 2014.

[8] D. Il'yasova, F. Wang, I. Spasojevic, K. Base, R. B. D’Agostino Jr., and L. E. Wagenknecht, "Urinary F2-isoprostanes, obesity, and weight gain in the IRAS cohort," Obesity (Silver Spring, Md.), vol. 20, no. 9, pp. 1915-1921, 1915.

[9] D. Il'yasova, I. Spasojevic, K. Base et al., "Urinary F2-isoprostanes as a biomarker of reduced risk of type 2 diabetes," Diabetes Care, vol. 35, no. 1, pp. 173-174, 2012.

[10] S. Basu, "F2-isoprostanes in human health and diseases: from molecular mechanisms to clinical implications," Antioxidants \& Redox Signaling, vol. 10, no. 8, pp. 1405-1434, 2008.

[11] T. Dorjgochoo, Y.-T. Gao, W.-H. Chow et al., "Major metabolite of $\mathrm{F}_{2}$-isoprostane in urine may be a more sensitive biomarker of oxidative stress than isoprostane itself," The American Journal of Clinical Nutrition, vol. 96, no. 2, pp. 405-414, 2012.

[12] D. Il'Yasova, F. Wang, I. Spasojevic, K. Base, R. B. D’Agostino Jr., and L. E. Wagenknecht, "Racial differences in urinary F2-isoprostane levels and the cross-sectional association with BMI," Obesity, vol. 20, no. 10, pp. 2147-2150, 2012.

[13] D. Il'Yasova, I. Spasojevic, F. Wang et al., "Urinary biomarkers of oxidative status in a clinical model of oxidative assault," Cancer Epidemiology Biomarkers \& Prevention, vol. 19, no. 6, pp. 15061510, 2010.

[14] M. B. Kadiiska, B. C. Gladen, D. D. Baird et al., "Biomarkers of oxidative stress study II: are oxidation products of lipids, proteins, and DNA markers of $\mathrm{CCl} 4$ poisoning?" Free Radical Biology \& Medicine, vol. 38, no. 6, pp. 698-710, 2005.

[15] T. R. Figueira, M. H. Barros, A. A. Camargo et al., "Mitochondria as a source of reactive oxygen and nitrogen species: from molecular mechanisms to human health," Antioxidants \& Redox Signaling, vol. 18, no. 16, pp. 2029-2074, 2013.

[16] J. P. DeLany, J. J. Dubé, R. A. Standley et al., "Racial differences in peripheral insulin sensitivity and mitochondrial capacity in the absence of obesity," The Journal of Clinical Endocrinology \& Metabolism, vol. 99, no. 11, pp. 4307-4314, 2014.

[17] B. Sirikul, B. A. Gower, G. R. Hunter, D. E. Larson-Meyer, and B. R. Newcomer, "Relationship between insulin sensitivity and in vivo mitochondrial function in skeletal muscle," American Journal of Physiology - Endocrinology and Metabolism, vol. 291, no. 4, pp. E724-E728, 2006.

[18] R. N. Cortright, K. M. Sandhoff, J. L. Basilio et al., "Skeletal muscle fat oxidation is increased in African-American and white women after 10 days of endurance exercise training," Obesity, vol. 14, no. 7, pp. 1201-1210, 2006.

[19] G. R. Hunter, R. L. Weinsier, J. P. McCarthy, D. Enette LarsonMeyer, and B. R. Newcomer, "Hemoglobin, muscle oxidative capacity, and VO2max in African-American and Caucasian women," Medicine and Science in Sports and Exercise, vol. 33, no. 10, pp. 1739-1743, 2001.

[20] K. Bryc, A. Auton, M. R. Nelson et al., "Genome-wide patterns of population structure and admixture in West Africans and African Americans," Proceedings of the National Academy of Sciences of the United States of America, vol. 107, no. 2, pp. 786791, 2010.

[21] R. Yaeger, A. Avila-Bront, K. Abdul et al., "Comparing genetic ancestry and self-described race in African Americans born in the United States and in Africa," Cancer Epidemiology, Biomarkers \& Prevention, vol. 17, no. 6, pp. 1329-1338, 2008.

[22] G. L. Milne, H. Yin, J. D. Brooks, S. Sanchez, L. Jackson Roberts II, and J. D. Morrow, "Quantification of F2-isoprostanes in biological fluids and tissues as a measure of oxidant stress," Methods in Enzymology, vol. 433, pp. 113-126, 2007.

[23] S. Lakkur, R. M. Bostick, D. Roblin et al., "Oxidative balance score and oxidative stress biomarkers in a study of Whites, African Americans, and African immigrants," Biomarkers, vol. 19, no. 6, pp. 471-480, 2014.

[24] M. Gross, M. Steffes, D. R. Jacobs Jr. et al., "Plasma $F_{2}$-isoprostanes and coronary artery calcification: the CARDIA Study," Clinical Chemistry, vol. 51, no. 1, pp. 125-131, 2005.

[25] A. M. Kanaya, C. L. Wassel, P. J. Stoddard et al., "F 2isoprostanes and adiposity in older adults," Obesity, vol. 19, no. 4, pp. 861-867, 2011.

[26] A. J. Fiocco, A. M. Kanaya, K. M. Lindquist et al., "Plasma F2isoprostane level and cognitive function over eight years in nondemented older adults: findings from the Health ABC Study," Prostaglandins Leukotrienes and Essential Fatty Acids, vol. 84, no. 1-2, pp. 57-61, 2011.

[27] A. O. Odegaard, D. R. Jacobs, O. A. Sanchez, D. C. Goff, A. P. Reiner, and M. D. Gross, "Oxidative stress, inflammation, endothelial dysfunction and incidence of type 2 diabetes," Cardiovascular Diabetology, vol. 15, no. 1, article 51, 2016.

[28] S. R. Colberg, R. J. Sigal, B. Fernhall et al., "Exercise and type 2 diabetes: the American College of Sports Medicine and the American Diabetes Association: joint position statement," Diabetes Care, vol. 33, no. 12, pp. e147-e167, 2010. 
[29] M. G. Nikolaidis, A. Kyparos, and I. S. Vrabas, "F $F_{2}$-isoprostane formation, measurement and interpretation: the role of exercise," Progress in Lipid Research, vol. 50, no. 1, pp. 89-103, 2011.

[30] L. W. Jones, N. D. Eves, I. Spasojevic, F. Wang, and D. Ilyasova, "Effects of aerobic training on oxidative status in postsurgical non-small cell lung cancer patients: A Pilot Study," Lung Cancer, vol. 72, no. 1, pp. 45-51, 2011.

[31] D. Il'yasova, J. D. Morrow, and L. E. Wagenknecht, "Urinary $\mathrm{F}_{2}$-isoprostanes are not associated with increased risk of type 2 diabetes," Obesity Research, vol. 13, no. 9, pp. 1638-1644, 2005.

[32] D. Il'yasova, L. E. Wagenknecht, I. Spasojevic et al., "Urinary F2isoprostanes and metabolic markers of fat oxidation," Oxidative Medicine and Cellular Longevity, vol. 2015, Article ID 729191, 5 pages, 2015.

[33] C. Weyer, R. E. Pratley, A. D. Salbe, C. Bogardus, E. Ravussin, and P. A. Tataranni, "Energy expenditure, fat oxidation, and body weight regulation: a study of metabolic adaptation to long-term weight change," Journal of Clinical Endocrinology and Metabolism, vol. 85, no. 3, pp. 1087-1094, 2000.

[34] S. A. Akohoue, S. Shankar, G. L. Milne et al., "Energy expenditure, inflammation, and oxidative stress in steady-state adolescents with sickle cell anemia," Pediatric Research, vol. 61, no. 2, pp. 233-238, 2007.

[35] J. M. Hibbert, L. L. Hsu, S. J. Bhathena et al., "Proinflammatory cytokines and the hypermetabolism of children with sickle cell disease," Experimental Biology and Medicine, vol. 230, no. 1, pp. 68-74, 2005.

[36] D. B. Allison, J. E. Gomez, S. Heshka et al., "Decreased resting metabolic rate among persons with Down Syndrome," International Journal of Obesity, vol. 19, no. 12, pp. 858-861, 1995.

[37] A. A. Tolun, P. M. Scarbrough, H. Zhang et al., "Systemic oxidative stress, as measured by urinary allantoin and $\mathrm{F}_{2}$ isoprostanes, is not increased in down syndrome," Annals of Epidemiology, vol. 22, no. 12, pp. 892-894, 2012.

[38] K. Broedbaek, R. Ribel-Madsen, T. Henriksen et al., "Genetic and environmental influences on oxidative damage assessed in elderly Danish twins," Free Radical Biology \& Medicine, vol. 50, no. 11, pp. 1488-1491, 2011.

[39] C. E. Fealy, A. Mulya, and J. P. Kirwan, "Lifestyle intervention reduces skeletal muscle dynamin-related protein-1 (Drp1) activation in obese insulin resistant humans," The FASEB Journal, vol. 27, supplement 1, pp. 1209-1218, 2013.

[40] M. J. Gibala and S. L. McGee, "Metabolic adaptations to shortterm high-intensity interval training: a little pain for a lot of gain?" Exercise and Sport Sciences Reviews, vol. 36, no. 2, pp. 58-63, 2008.

[41] N. Fillmore and G. D. Lopaschuk, "Targeting mitochondrial oxidative metabolism as an approach to treat heart failure," Biochimica et Biophysica Acta (BBA)-Molecular Cell Research, vol. 1833, no. 4, pp. 857-865, 2013.

[42] L. K. Williams, B. Padhukasahasram, B. K. Ahmedani et al., "Differing effects of metformin on glycemic control by raceethnicity," Journal of Clinical Endocrinology and Metabolism, vol. 99, no. 9, pp. 3160-3168, 2014.

[43] G. Davì, M. T. Guagnano, G. Ciabattoni et al., "Platelet activation in obese women: role of inflammation and oxidant stress," The Journal of the American Medical Association, vol. 288, no. 16, pp. 2008-2014, 2002.

[44] M. Rosenbaum and R. L. Leibel, "Models of energy homeostasis in response to maintenance of reduced body weight," Obesity, vol. 24, no. 8, pp. 1620-1629, 2016.
[45] C. Bouchard and A. Tremblay, "Genetic influences on the response of body fat and fat distribution to positive and negative energy balances in human identical twins," Journal of Nutrition, vol. 127, no. 5, supplement, pp. 943s-947s, 1997.

[46] P. S. MacLean, R. R. Wing, T. Davidson et al., "NIH working group report: innovative research to improve maintenance of weight loss," Obesity, vol. 23, no. 1, pp. 7-15, 2015. 


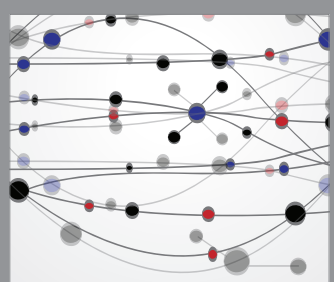

The Scientific World Journal
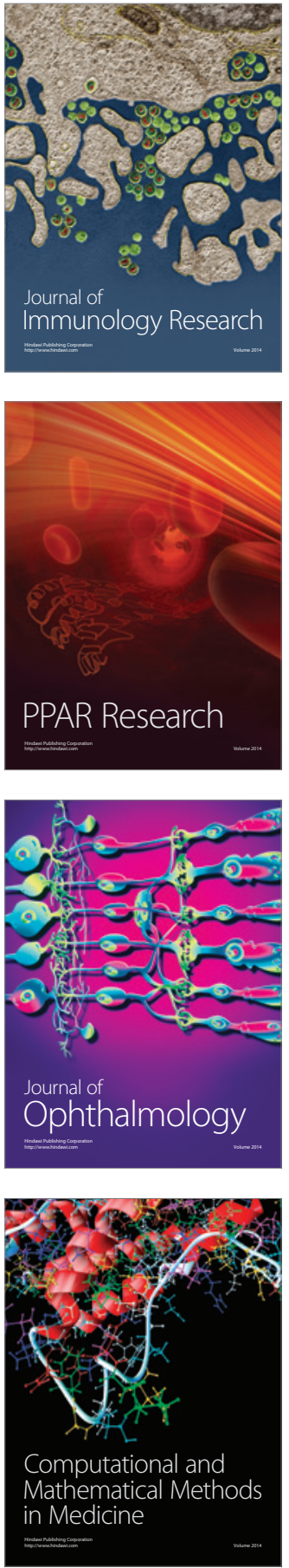

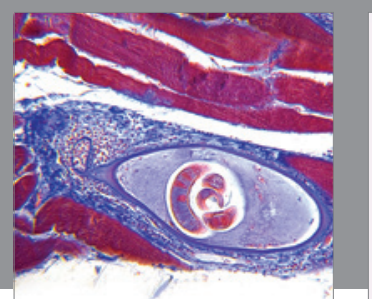

Gastroenterology Research and Practice
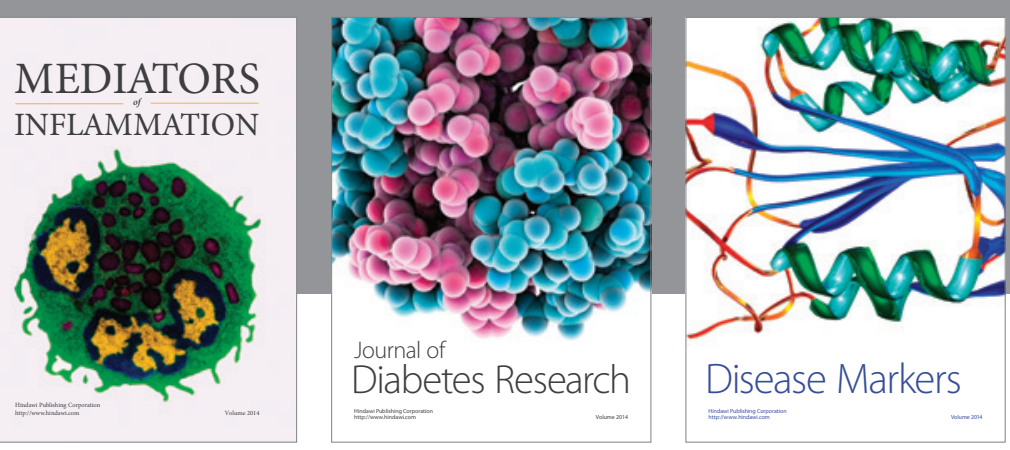

Disease Markers

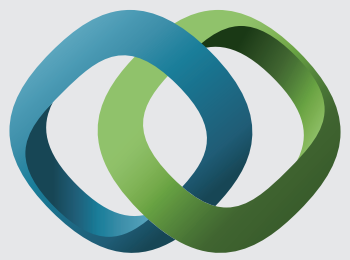

\section{Hindawi}

Submit your manuscripts at

https://www.hindawi.com
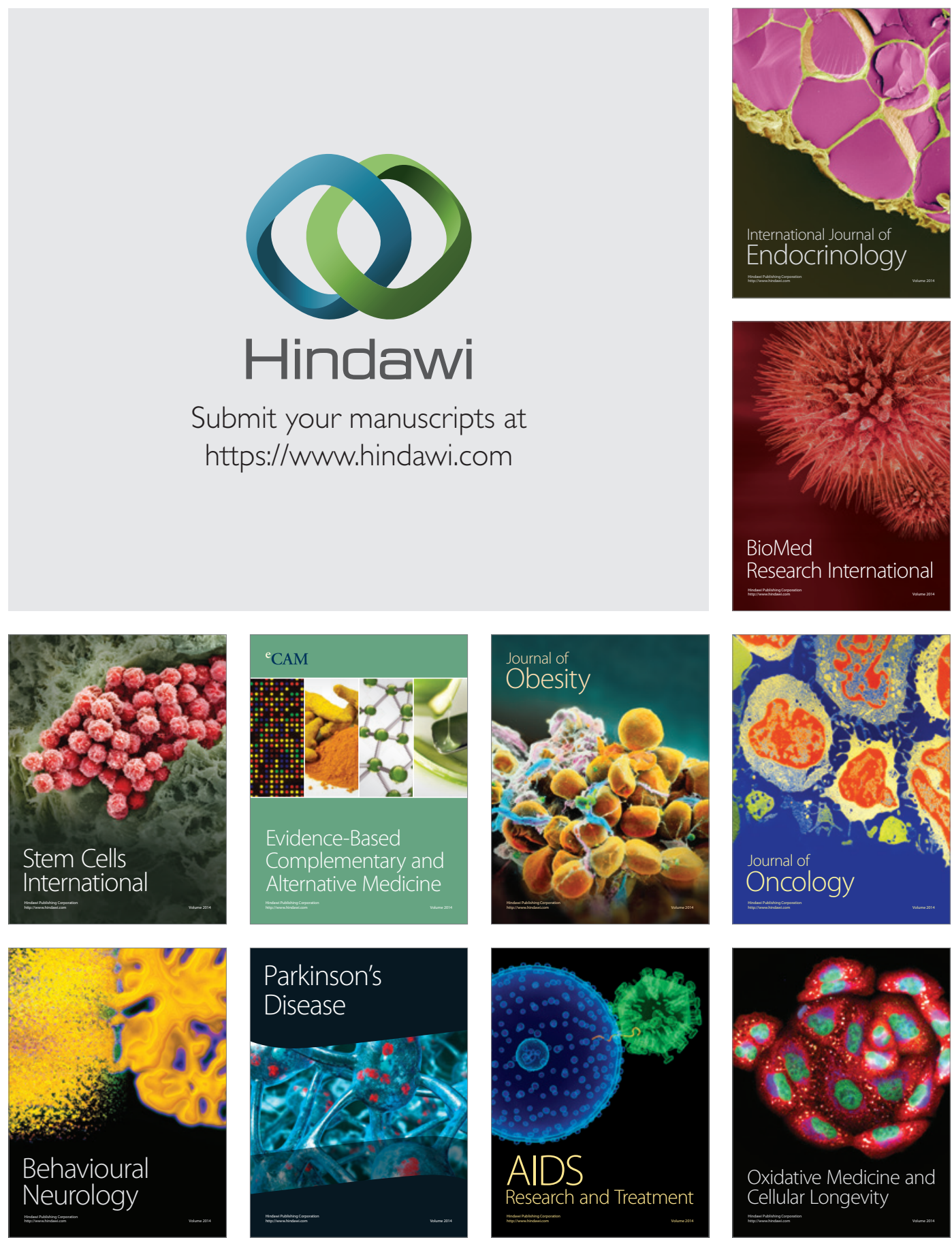